\title{
Infografías en el aprendizaje del cálculo en educación básica
}

\section{Computer graphics in the learning of calculus in basic education}

\author{
Carlos Sebastián Gómez-Vergel ${ }^{1}$ \\ Mawency Vergel-Ortega ${ }^{2}$ \\ Luisa Stella Paz-Montes ${ }^{3}$ \\ Universidad de Los Andes, Bogotá- Colombia \\ Universidad Francisco de Paula Santander, \\ Norte de Santander, Cúcuta-Colombia
}

\section{RESUMEN}

La investigación tiene por objeto analizar la incidencia de los gráficos digitales en el aprendizaje del cálculo. Sigue un enfoque cuantitativo con apoyo cualitativo mediante el uso de elementos del ciberperiodismo y a partir de métodos cuantitativos. El proceso se desarrolló en instituciones de educación básica de Ocaña y Tibú (Colombia), con un $1 . \quad$ Ingeniería Electrónica (Estudiante). Universidad de Los Andes-Colombia. cs.gomezv@uniandes.edu.co orcid: https://orcid.org/0000-0002-6176-3613

1. 2 Doctora en Educación. Postdoctora en Imaginarios y representaciones sociales. Docente y Directora del Departamento de Matemáticas y Estadística. Filiación: Universidad Francisco de Paula Santander. Correo electrónico: mawency@ufps.edu.co. Orcid: https://orcid. org/0000-0001-8285-2968

$1.3 \quad$ Doctora en Educación. Filiación: Universidad Francisco de Paula Santander Cúcuta - Colombia correo: luisastellapm@ufps.edu.co-Ipazmontes@gmail.com Orcid: https://orcid.org/0000-0002-8887-3441 grupo de estudiantes en edades entre 10 y 15 años de edad. El objetivo del proyecto fue diseñar y aplicar una estrategia pedagógica para el desarrollo de la competencia en la resolución de problemas de cálculo basada en el ciberperiodismo y diseño de gráficos digitales por computadora. Los resultados demostraron que infografías y proyectos en comunicación del conocimiento contribuye a la conceptualización en temas de cálculo, a desarrollar habilidades de comunicación en hipertextualidad, diseño, creatividad, interactividad, al mejoramiento de las habilidades en variedad, profundidad, redacción de textos y en el desarrollo de la competencia de interpretación de problemas.

\section{ABSTRACT.}

The research aims to analyze the incidence of digital graphics in the learning of calculus, 
following a quantitative approach with qualitative support through the use of elements of cyberjournalism and from quantitative methods. The process was developed in basic education institutions in Ocaña and Tibú (Colombia), with a group of students between 10 and 15 years old. The objective of the project was to design and apply a pedagogical strategy for the development of competence in the resolution of calculation problems based on cyberjournalism and digital computer graphics design. The results showed that computer graphics and knowledge communication projects contribute to the conceptualization of calculation issues, to the development of communication skills in hypertextuality, design, creativity, interactivity, to the improvement of skills in variety, depth, text writing and in the development of problem interpretation competence.

\section{INTRODUCCIÓN}

Transformaciones actuales de procesos de comunicación desde el uso de correos, videoconferencias, web, chat, periódicos electrónicos, productos on-line han ampliado posibilidades de desarrollar competencias en la juventud a partir de metodologías donde confluyan convenciones narrativas[1]. La inclusión de estas prácticas en la enseñanza del cálculo, lleva a replantear modelos y herramientas que incorporen las etapas del proceso comunicativo como apoyo del aprendizaje [2]. En este sentido, abordar el proceso de enseñanza desde la perspectiva de la interpretación de la resolución de problemas [3], incorporando elementos informativos de tipo audiovisual, puede contribuir a captar el interés de los jóvenes y, por lo tanto, permitir que el proceso de aprendizaje sea significativo [2]. Dificultades centradas en la interpretación y desarrollo del proceso en el aula o a través de medios sincrónicos o asincrónicos donde se limita a señalar un problema y proporcionar soluciones basadas en procedimientos repetitivos[4]. En este sentido, una de las principales carencias que siguen presentando los alumnos es la que tiene que ver con la resolución de problemas en el contexto [5]. En cálculo, los estudiantes utilizan aún datos sin mostrar una clara comprensión de los mismos y sin identificar relaciones operativas, conceptuales o procedimentales, soluciones que a veces son válidas pero que algunas veces carecen de estrategia cognitiva y de pensamiento crítico [6]. Por otro lado, los estudios muestran que los estudiantes logran una mayor retención al presentar elementos visuales y le motivan a profundizar información [7]. El análisis de la incidencia de la aplicación de herramientas como las infografías, permite evidenciar si el estudiante va más allá de la operacionalización.

\section{MÉTODO}

El proceso de investigación se desarrolló a través del enfoque mixto, desde lo cuantitativo [8] guiado por conceptos empíricos medibles [9], con un diseño es cuasi-experimental, en el que se examinan las relaciones de causa y efecto entre variables independientes y dependientes [10], se relaciona sin modificarlas intencionadamente [11], se propuso el uso del método de cuatro grupos de Salomón [12]. Para el desarrollo de este método, el número total de sujetos del estudio se asignó aleatoriamente a los cuatro grupos; en el primer grupo se hizo una medición previa de la variable dependiente, luego se aplicó el tratamiento experimental y finalmente se hizo una medición posterior; en el segundo grupo se hicieron mediciones previas y posteriores sin aplicar el tratamiento experimental; el tercer grupo recibió el tratamiento experimental y se hizo una medición posterior; y finalmente, en el cuarto grupo sólo se hizo una medición posterior [13]. Una vez formados los cuatro grupos y realizado el proceso de intervención y evaluación, se establecen las relaciones entre la información recogida, proceso que es complejo desde el punto de vista estadístico 
[14]. La muestra aleatoria está compuesta por 120 jóvenes de 10 a 15 años de edad en Ocaña y Tibú, Colombia.

La investigación se realizó en tres fases. En la primera fase se realizó una caracterización, seguido de test y posicionamiento de estudiantes acorde a teoría de respuesta al item, cuyo objetivo fue hacer un diagnóstico del nivel de competencia interpretativa de los estudiantes en la resolución de problemas, se calculó el factor Anova, con un nivel de confianza del $95 \%$. Se generaron dos contextualizados para el fomento en pruebas tipo, con preguntas de opción múltiple de una sola respuesta, que fueron escaneadas y puntuadas con una versión de prueba de un software de reconocimiento de marca, que facilita la puntuación de las pruebas. En la segunda, se diseñó y aplicó una estrategia pedagógica para fortalecer la solución de problemas en el contexto. Por último, en la tercera fase, se aplicó un post-test para medir la eficacia de la propuesta implementada, específicamente en el concurso de resolución de problemas. En la etapa cualitativa, de acuerdo al análisis de contenido y al método fenomenológico, se le hicieron preguntas al estudiante [15] para que interprete el enunciado, con el propósito de que los elementos del problema se presenten y como estos se interrelacionan, en la segunda etapa, la configuración de un plan, el estudiante debe pensar en la estrategia más adecuada para resolver el problema planteado, por lo que se le presentan una serie de estrategias para que de acuerdo al problema elija la más adecuada [16], seguido de la resolución del modelo planteado [17]. En la última fase crítica, se verifica si la solución cumple con los requisitos del problema [18].

\section{RESULTADOS}

Aplicando el pre-test a dos grupos A y B de 30 estudiantes cada uno, nos permitió entender que los resultados encontrados en ellos son similares. Encontramos que el promedio del grupo A fue de 3,4 y el del grupo B de 3,5 de 10 puntos. Además, las desviaciones estándar con valores de 0,428 y 0,713 respectivamente. La prueba de chi cuadrado, $p>0,05$ indicó que la prueba y la variable del grupo en A, B son independientes, es decir, los resultados no dependieron de la forma en que se seleccionaron los grupos, asi mismo, prueba Smirnov-Kolmogorov $p>0,05$, lo cual indicó que los grupos estaban distribuidos normalmente. En la prueba de homogeneidad de las varianzas con la estadística de Levesne $p>0,05$ suponiendo que las varianzas son las mismas para los grupos. Una vez probados estos supuestos, se realiza una prueba " $t$ " para comparar las medias con $p>0,05$, entonces no hay pruebas para pensar que los grupos difieren entre sí. Los grupos comienzan en condiciones similares (anova $=0.21$ ), valor $\mathrm{p}$ superior a 0,05 , lo que indica que las medias pueden considerarse similares. Una vez implementada la herramienta, los resultados de la prueba final, evaluados los cuatro grupos permiten observar que el promedio del grupo A fue de 9.2, mientras que el grupo B obtuvo 6.5 puntos de promedio. Del mismo modo, el grupo $C$ alcanzó un promedio de 7.32 y el grupo D 8.5. Esto implica que el grupo A, que realizó el pre-test y el post-test y la intervención, fue el grupo que obtuvo el mejor promedio, seguido por el grupo $D$ que tuvo la intervención, pero sólo presentó el post-test. Sin embargo, es posible afirmar que hay una mejora, ya que el aspecto en el que el grupo A avanzó más fue en la interpretación y formulación de nuevos problemas, en diseño y manejo de herramientas, y en las concepciones de aplicaciones de función ingreso, utilidad, costo, marginales, punto de equilibrio, amortizaciones, que fueron objeto de la investigación. Asimismo, la mayor fuerza del grupo $D$ fue planteamiento de estrategias de solución (media 8.9), lo que implica que se concentran en la planeación, pero bajo en interpretación. Esto significa que los conocimientos adquiridos por el grupo D se centraron en la habilidad, aunque logran el objetivo y resultado del aprendizaje esperado. 
Al analizar el grupo C, un grupo en el que no se realizó ninguna intervención ni ninguna prueba posterior, la media más alta por proceso se centró en diseño (media 8,5), incidencia de la prueba previa en la solución de la prueba posterior. Se puede observar que la intervención y la aplicación de infografías están asociadas al diseño, concepción, la interpretación del problema , la ejecución del plan para resolver el problema. Con un nivel de significación de 0,0029, el análisis de correlación bivariante muestra la correlación entre las variables de la prueba, la interpretación del problema y las habilidades $(0,6)$. Se puede afirmar que la metodología de implementando infografías y procesos de ciberperiodismo contribuyó a reconocimiento y acción interpretativa de los problemas, así como a la comunicación de resultados.

Frente al análisis cualitativo, surgen categorías asociadas de lógica, jerarquización de información, redacción de problemas, interpretación de resultados, aplicaciones, razón de cambio, análisis, contexto, modelo, ley, hipótesis. Estructura que guía el desarrollo del problema a través de la estructura del concepto, definición, procedimiento, con representaciones numéricas, simbólicas; alrededor de la fenomenología se determinan fenómenos como cálculos pendientes, contexto matemático, subestructura de aplicación de límites, aplicación de derivaciones, reglas máximas y mínimas, situación científica. Desde el ciberperiodismo, el análisis permite asociar categorías con la hipertextualidad, creatividad, actitud, reflexión, interacción, documentación, recuperación de información, búsqueda de soluciones, análisis de textos, razonamiento, como claves del elemento de desarrollo; la tecnología como elemento característico genera innovación desde un enfoque conceptual ligado a la comunicación.
La categoría de medio y digital surge de la observación de estudiantes cuyo rendimiento se lleva a cabo mediante infografías de diversa calidad, proposición de problemas en contexto, compartidos a través de la red, que poseen conocimientos y habilidades con diferente nivel de profundidad y enfoque. Por último, surge la función de lector-periodista, que articulan estrategias de formación asociada a habilidades que genera producción utilizando recursos de fuentes, telecomunicaciones, aplicación real y creación de roles, donde todos pueden transmitir información de manera creativa o analizar resultados de manera autónoma.

El desarrollo de competencias sitúa la infografía como herramienta de trabajo que mejora la información visual, conceptual, mejora el aprendizaje y práctica en temas de administración, de la autoformación, constituyéndose en herramienta didáctica en cálculo. Asimismo, al determinar la importancia de la influencia, el grado de interés de los estudiantes, la adopción de esta rutina de fomentó el autoaprendizaje, el trabajo en equipo, la generación y comunicación de ideas, comprensión lectora y fluidez verbal, y su valor pedagógico, como elemento que potencia las habilidades de comunicación y generación de contenidos, se reflejan en la mejor comprensión de conceptos, procedimientos, que finalmente se traducen en una mejora de los resultados de aprendizaje de los estudiantes. Finalmente, se puede afirmar que, al iniciar los grupos en un nivel de competencia similar, permite establecer si el proceso de intervención que se diseñó y ejecutó dio los resultados esperados. Asimismo, al tener cuatro grupos en el post-test, se verifica el nivel de incidencia que tiene la presentación del pre-test, así como el de la estrategia didáctica.

\section{CONCLUSIONES}

Infografías mejoran la conceptualización e interpretación de los problemas relacionados 
con el cálculo y matemática financiera en los estudiantes de educación básica.

Las categorías asociadas al cálculo y el plan para resolver problemas en administración, se asocian a procedimientos y concepciones en razón de cambio, movimientos, funciones, ecuaciones matemáticas, punto de equilibrio. La estrategia didáctica mejora la estructura que guía el desarrollo del problema a través de la concepción, definición, procedimiento, representaciones numéricas, gráficas, simbólicas; determina fenómenos como cálculos pendientes, contexto matemático, subestructura de aplicación de límites, aplicación de derivadas, reglas máximas y mínimas, vinculadas a una situación científica.

Las categorías asociadas a la interpretación y conceptualización en jóvenes con edades entre 10 y 15 años son creatividad, manejo de hipertexto, la inmediatez, la interactividad; infografía fomentó el autoaprendizaje, el trabajo en equipo, la comunicación de ideas, comprensión lectora y fluidez verbal, y su valor pedagógico como elemento que potencia las habilidades de comunicación y generación de contenidos, mejora habilidades en variedad, profundidad y enfoque del texto escrito al interpretar y la función lector-creador-actor.

\section{REFERENCIAS BIBLIOGRÁFICAS}

[1] J. Cabero Almenara, y M. Llorent, "La alfabetización digital de los alumnos, competencias digitales para el siglo XXI," Revista portuguesa de pedagogía, vol 42 no. 2 , p. 7-28, 2008.

[2] Duarte Y, Sánchez-Frank J, Gómez C 2019 El trabajo colaborativo en las competencias en estudiantes del Instituto Agrícola Región del Catatumbo-Colombia Covalente 136
[3] P. Carlino, "Alfabetización académica diez años después," Revista Mexicana de Investigación Educativa, vol. 38, no. 57, pp. 355381, 2013.

[4] B. Figueroa, M. Aillon y A. Fuentealba. "La escritura académica con soporte de esquemas digitales en la formación docente". Revista de Universidad y Sociedad del Conocimiento (RUSC). vol. 11 no. 1, 18-32, 2014 doi: $\underline{10.7238 /}$ rusc.v11i1.1665

[5] Rolls-Royce plc, The jet engine., 5th ed. Wiley, 2015.

[6] A. Ortega, M. Vergel y J. Martinez, "Validity of microrubri, instrument to measure the development of competences in mathematics Journal of Physics: Conference Series 1160 p. 125.

[7] H. Parra-López, J. Rojas-Suarez and M. Vergel-Ortega, "Curricular trends in the Universidad Francisco de Paula Santander academic program offerings," Journal of Physics: conferences series vol. 1329 no. 3201 p. 9, 2019.

[8] M. Vergel, J. Martínez and S. Zafra, "Apps en el rendimiento académico y auto concepto de estudiantes de ingeniería", Revista Logos Ciencia \& Tecnología vol. 6 no. 1, p. 98, 2015.

[9] M. Fujioka, "U.S. writing center theory and practice: implications for writing centers in Japanese universities", Kinki University Center for Liberal Arts and Foreign Languages Education Journal, vol. 2 no. 1, pp. 205 - 224, 2011.

[10] K. Guzmán Tinajero y S. Rojas-Drummond, "Escritura colaborativa en alumnos de primaria: un modelo social de aprender juntos," Revista mexicana de investigación educativa, vol. 17, no. 52, pp. 217-245, 2012. 
[11] G. Hays, "Learners helping learners in an EFL writing center", JALT2009 Conference Proceedings, vol. 17 no. 1, 2014.

[12] A. J. Stepanoff, Centrifugal and axial flow pumps. Theory, design and application. Wiley, 1957

[13] B. Tan, "Innovating writing centers and online writing labs outside North America," The Asian EFL Journal Quarterly, vol. 13, no. 2, pp. 390-417, 2015.

[14] S. Vivant, "Digital technology as a tool for the democratization of powerful mathematical ideas," Revista Colombiana de Educación, vol. 74 no. 1 , p. 10, 2018.

[15] J. Nieto, J. Rojas and M. Vergel. Impacto de estrategia pedagógica basada en el aprendizaje creativo para estudiantes de ingeniería. Editorial Ibáñez, 2019

[16] Rojas, J., Vergel, M. y Urbina, J. (2019). Imaginarios de educación superior en la juventud sorda. Bogota: ECOE Ediciones

[17] Vergel-Ortega, M., Gómez-Vergel, C. S., \& Caravalho-Casanova, J. F. (2020). Cuentos sobre las emociones de jóvenes universitarios durante el proceso de aprendizaje del cálculo en ingeniería. Respuestas, 25(1), 89-95. https://doi. org/10.22463/0122820X.2414

[18] Z. Bauman, Z. Mundo consumo. Barcelona: Paidós. 2010. 